PROCEEDINGS OF THE AMERICAN MATHEMATICAL SOCIETY

Volume 124, Number 8, August 1996

\title{
INTEGRAL EQUATIONS, IMPLICIT FUNCTIONS, AND FIXED POINTS
}

\author{
T. A. BURTON
}

(Communicated by Hal L. Smith)

\begin{abstract}
The problem is to show that (1) $V(t, x)=S\left(t, \int_{0}^{t} H(t, s, x(s)) d s\right)$ has a solution, where $V$ defines a contraction, $\tilde{V}$, and $S$ defines a compact map, $\tilde{S}$. A fixed point of $P \varphi=\tilde{S} \varphi+(I-\tilde{V}) \varphi$ would solve the problem. Such equations arise naturally in the search for a solution of $f(t, x)=0$ where $f(0,0)=0$, but $\partial f(0,0) / \partial x=0$ so that the standard conditions of the implicit function theorem fail. Now $P \varphi=\tilde{S} \varphi+(I-\tilde{V}) \varphi$ would be in the form for a classical fixed point theorem of Krasnoselskii if $I-\tilde{V}$ were a contraction. But $I-\tilde{V}$ fails to be a contraction for precisely the same reasons that the implicit function theorem fails. We verify that $I-\tilde{V}$ has enough properties that an extension of Krasnoselskii's theorem still holds and, hence, (1) has a solution. This substantially improves the classical implicit function theorem and proves that a general class of integral equations has a solution.
\end{abstract}

\section{INTRODUCTION}

Motivated by a problem in implicit function theory, we formulate an existence problem in integral equations. Two fixed point theorems are proved enabling us to solve the integral equation. The work can be introduced in three subsections.

(a) The problem: An integral equation. Equations of the form

$$
V(t, x)=S\left(t, \int_{0}^{t} H(t, s, x(s)) d s\right)
$$

arise in a natural way. It is supposed that there is an $\alpha>0$ such that $V, S$ : $[-\alpha, \alpha] \times[-\alpha, \alpha] \rightarrow R$ and $H:[-\alpha, \alpha] \times[-\alpha, \alpha] \times[-\alpha, \alpha] \rightarrow R$ are continuous and $S(0,0)=V(t, 0)=0$. The problem is to find a $\beta>0$ and a function $\varphi$ : $[-\beta, \beta] \rightarrow R, \varphi(0)=0$, such that $x=\varphi(t)$ satisfies (1). By changes of variable, many equations will fit this form. The general theory for $(1)$ when $V(t, x)=x$ and $S$ is linear is found in Corduneanu [1].

In the present problem $V$ will define a contraction $\tilde{V}$ on the complete metric space of continuous $\psi:[-\beta, \beta] \rightarrow R$ with the supremum metric, while $S$ will define a compact mapping, $\tilde{S}$. Thus, we will seek a fixed point of the mapping $P \varphi=\tilde{S} \varphi+(I-\tilde{V}) \varphi$. We use the terminology of Smart [8, p. 25] to say that a

Received by the editors February 6, 1995.

1991 Mathematics Subject Classification. Primary 45D05, 26B10, 47H10.

Key words and phrases. Integral equations, implicit functions, fixed points.

(C)1996 American Mathematical Society 
mapping is compact if it maps a set $M$ in a topological space $X$ into a compact subset of $X$.

(b) Motivation: Implicit functions. The simplest example concerns the standard implicit function theorem. Given the scalar equation

$$
f(t, x)=0 \text { with } f(0,0)=0,
$$

the classical problem is to find a $\beta>0$ and a continuous function $\varphi:[-\beta, \beta] \rightarrow R$, $\varphi(0)=0$, such that $f(t, \varphi(t)) \equiv 0$; in other words, can we solve $f(t, x)=0$ for $x=\varphi(t)$ ?

There are three classical attacks on the problem: one can use techniques of advanced calculus (cf. Taylor and Mann [9, pp. 225-232]), fixed point theory (cf. Smart [8, p. 6]), and differential equations (cf. Hartman [2, pp. 5, 11-12]), under the common assumption that

$$
\frac{\partial f}{\partial x}(0,0) \neq 0
$$

The intuitive reason for (3) is clear. If $f$ is differentiable, then it can be approximated arbitrarily well by a linear function near $(0,0)$ so that $z=f(t, x)$ can be approximated by a plane intersecting the plane $z=0$. The precise statement is given as follows from Smart [8, p. 6]; an $n$-dimensional analog with parameters can be found in Hartman [2, p. 5].

Theorem (Implicit function). Let $N$ be a neighborhood of $(0,0)$ in which $f: N \rightarrow$ $R$ is continuous, $\partial f / \partial x$ exists in $N$ and is continuous at $(0,0), \partial f(0,0) / \partial x \neq 0$, and $f(0,0)=0$. Then there is a unique continuous function $\varphi$ with $f(t, \varphi(t))=0$.

Condition (3) allows one to construct a contraction mapping on a complete metric space with a fixed point $\varphi$. In the same way, when (3) holds we can reverse the following steps to obtain $\varphi$. We have from (2) that

$$
\frac{\partial f}{\partial t}(t, x)+\frac{\partial f(t, x)}{\partial x} \frac{d x}{d t}=0
$$

and for $(t, x)$ near $(0,0)$, then

$$
\frac{d x}{d t}=-(\partial f(t, x) / \partial t) /(\partial f(t, x) / \partial x)=: G(t, x),
$$

so with $x(0)=0$ we obtain

$$
x(t)=\int_{0}^{t} G(s, x(s)) d s .
$$

If $G$ is continuous, then (6) has a solution $\varphi$ by the Peano existence theorem (cf. Hartman [2, p. 10] or Smart [8, p. 44]).

On the other hand, if (3) fails, then we write (4) as

$$
P(t, x) x^{\prime}=F(t, x), \quad P(0,0)=0,
$$

where we emphasize that $P$ is not necessarily $\partial f / \partial x$. We can invert that differential operator in (7) by writing

$$
\int_{0}^{x} P_{t}(t, s) d s+P(t, x) x^{\prime}=F(t, x)+\int_{0}^{x} P_{t}(t, s) d s
$$


or

$$
\frac{d}{d t} \int_{0}^{x} P(t, s) d s=F(t, x)+\int_{0}^{x} P_{t}(t, s) d s,
$$

and so an integration and use of $x(0)=0$ yield

$$
\int_{0}^{x} P(t, s) d s=\int_{0}^{t}\left[F(s, x(s))+\int_{0}^{x(s)} P_{t}(s, v) d v\right] d s,
$$

which is a form of $(1)$ with $V(t, 0)=0$.

If we can prove that (8) has a solution and reverse the steps from (8) back to (2), then the problem is solved.

The components of (8) should be no surprise. Krasnoselskii (cf. Smart [8, p. 31]) studied Schauder's [6] work on partial differential equations and noticed that inversion of a perturbed differential operator yields a contraction and compact map.

Now our problem began with (2), and we should not neglect a chance which presents itself here. Even though $P(0,0)=0$, frequently (8) can be inverted by inspection so that (8) results in

$$
x(t)=S\left(t, \int_{0}^{t} H(s, x(s)) d s\right), \quad S(0,0)=0,
$$

which is readily solved by means of Schauder's theorem since $S$ defines a compact mapping. A simple example occurs when $P(t, x)=(t-x)^{2}$, and this type is treated in our subsequent Example 1.

But the general form of (8) can be formidable. It leads us to two fixed point theorems.

(c) A solution: Fixed point theorems. It turns out that a fixed point theorem of Krasnoselskii [3, p. 370] (cf. Smart [8, p. 31]) readily applies to (1) when $\int_{0}^{t} H(t, s, x(s)) d s$ defines a compact map and when $x-V(t, x)$ defines a contraction mapping. But it is the very property that $\partial f(0,0) / \partial x=0$ which frequently causes $x-V(t, x)$ to fail to define a contraction mapping.

The focus of the solution of (1) is then on how much we can weaken a contraction and still complete the details of Krasnoselskii's proof. In this paper we define a weaker contraction property on a mapping $B$. It then turns out that $(I-B)$ is a homeomorphism. A translate of $B$ still has a fixed point. Thus, Krasnoselskii's theorem still holds. This allows us to finish the problem and show that (1) has a solution. Two simple, but instructive, examples are given.

\section{TWO FIXED POINT THEOREMS}

If $\partial V(t, x) / \partial x=0$ at $(0,0)$ and is continuous, then there is a $\gamma<1$ with $|\partial V(t, x) / \partial x| \leq \gamma$ for $(t, x)$ small. Thus, $V$ will define a contraction mapping $\tilde{V}$ on the complete metric space $(X, \rho)$ of continuous functions $\varphi:[-\alpha, \alpha] \rightarrow R$ with $\rho(\varphi, \psi)=\sup _{t \in[-\alpha, \alpha]}|\varphi(t)-\psi(t)|$ for $\alpha, \varphi$, and $\psi$ small. We will have $\rho(\tilde{V} \varphi, \tilde{V} \psi) \leq$ $\gamma \rho(\varphi, \psi)$, where $(\tilde{V} \varphi)(t)=V(t, \varphi(t))$ for $-\alpha \leq t \leq \alpha$. Thus, the left-hand side of (1) will define a contraction mapping, while the right-hand side will map bounded sets into compact sets. We follow (as mentioned earlier) Smart's terminology and say that a mapping of a set into a compact set is a compact map. We think of (1) as $(P \varphi)(t)=(A \varphi)(t)-(\tilde{V} \varphi)(t)+\varphi(t)$. A fixed point will solve (1). Thus, we have a compact mapping $A$ and a mapping $B=I-\tilde{V}$. Now $B$ is not generally 
a contraction if $\partial V(0,0) / \partial x=0$; but if $\partial V / \partial x>0$ for $(t, x) \neq(0,0)$, then $B$ is a shrinking map. (If $\partial V / \partial x<0$ for $(t, x) \neq(0,0)$, then use $P \varphi=-A \varphi+\tilde{V} \varphi+I \varphi$.) In fact, it may be much more.

Definition. Let $(M, \rho)$ be a metric space and $B: M \rightarrow M$. $B$ is said to be a large contraction if $\rho(B \varphi, B \psi)<\rho(\varphi, \psi)$ for $\varphi, \psi \in M$, with $\varphi \neq \psi$, and if $\forall \varepsilon>0 \exists \delta<1$ such that $[\varphi, \psi \in M, \rho(\varphi, \psi) \geq \varepsilon] \Rightarrow \rho(B \varphi, B \psi) \leq \delta \rho(\varphi, \psi)$.

Theorem 1. Let $(M, \rho)$ be a complete metric space and $B$ a large contraction. Suppose there is an $x \in M$ and an $L>0$, such that $\rho\left(x, B^{n} x\right) \leq L$ for all $n \geq 1$. Then $B$ has a unique fixed point in $M$.

Proof. For that $x \in M$, consider $\left\{B^{n} x\right\}$. If this is a Cauchy sequence, then the standard argument (cf. Smart [8, pp. 1-3]) shows that the limit is a fixed point. By way of contradiction, if $\left\{B^{n} x\right\}$ is not a Cauchy sequence, then $\exists \varepsilon>0, \exists\left\{N_{k}\right\} \uparrow \infty$, $\exists n_{k}>N_{k}, \exists m_{k}>N_{k}, m_{k}>n_{k}$, with $\rho\left(B^{m_{k}} x, B^{n_{k}} x\right) \geq \varepsilon$. Thus,

$$
\begin{aligned}
\varepsilon & \leq \rho\left(B^{m_{k}} x, B^{n_{k}} x\right) \leq \rho\left(B^{m_{k}-1} x, B^{n_{k}-1} x\right) \\
& \leq \cdots \leq \rho\left(x, B^{m_{k}-n_{k}} x\right)
\end{aligned}
$$

since $B$ is a large contraction, for this $\varepsilon>0$ there is a $\delta<1$ with

$$
\begin{aligned}
\varepsilon \leq \rho\left(B^{m_{k}} x, B^{n_{k}} x\right) \leq \delta \rho\left(B^{m_{k}-1} x, B^{n_{k}-1} x\right) \\
\leq \cdots \leq \delta^{n_{k}} \rho\left(x, B^{m_{k}-n_{k}} x\right) \\
\quad \leq \delta^{n_{k}} L,
\end{aligned}
$$

a contradiction for large $n_{k}$ since $\delta<1$ and $\varepsilon>0$. This completes the proof.

Remark. Theorem 1 relates to an open question (cf. Smart [8, p. 39]). Does a shrinking map of the unit ball have a fixed point? It does if it is a large contraction.

Lemma. If $(S,\|\cdot\|)$ is a normed space, if $X \subset S$, and if $B: X \rightarrow S$ is a large contraction, then $(I-B)$ is a homeomorphism of $X$ onto $(I-B) X$.

Proof. Clearly, $I-B$ is continuous. To see that $(I-B)$ is $1-1$, if $x \neq y$, then

$$
\begin{aligned}
\|(I-B) x-(I-B) y\| & \geq\|x-y\|-\|B x-B y\| \\
& >\|x-y\|-\|x-y\|=0 .
\end{aligned}
$$

Hence, $(I-B)$ is $1-1$ and $(I-B)^{-1}$ exists.

Suppose that $(I-B)^{-1}$ is not continuous. Then $\exists(I-B) y$ and $(I-B) x_{n} \rightarrow$ $(I-B) y$, but $x_{n} \not y$. Now for each $\varepsilon>0 \exists N$ such that $n \geq N \Rightarrow$

$$
\varepsilon \geq\left\|(I-B) x_{n}-(I-B) y\right\| \geq\left\|x_{n}-y\right\|-\left\|B y-B x_{n}\right\| .
$$

Since $x_{n} \nrightarrow y, \exists \varepsilon_{0}>0$ and $\left\{x_{n_{k}}\right\}$ with $\left\|y-x_{n_{k}}\right\| \geq \varepsilon_{0}$; as $B$ is a large contraction, there is a $\delta<1$ with $\left\|B y-B x_{n_{k}}\right\| \leq \delta\left\|y-x_{n_{k}}\right\|$. Thus, from $(*)$ we have

$$
\begin{aligned}
\varepsilon \geq \| & (I-B) x_{n_{k}}-(I-B) y \| \\
& \geq\left\|x_{n_{k}}-y\right\|-\delta\left\|x_{n_{k}}-y\right\| \\
& =(1-\delta)\left\|x_{n_{k}}-y\right\| \\
& \geq(1-\delta) \varepsilon_{0} .
\end{aligned}
$$

But $\varepsilon_{0}$ is fixed, $\delta<1$, and a contradiction occurs as $\varepsilon \rightarrow 0$; that is, as $\varepsilon \rightarrow 0$, $n_{k} \rightarrow \infty$, but $\varepsilon_{0}$ remains fixed. This completes the proof. 
Theorem 2. Let $(S,\|\cdot\|)$ be a Banach space and $M$ a bounded, convex nonempty subset of $S$. Suppose that $A, B: M \rightarrow M$ and that

$$
x, y \in M \Rightarrow A x+B y \in M,
$$

$A$ is continuous and $A M$ is contained in a compact subset of $M$,

$$
B \text { is a large contraction. }
$$

Then $\exists y \in M$ with $A y+B y=y$.

Proof. For each fixed $y \in M$ the mapping $H z=B z+A y$ is a large contraction on $M$ with unique fixed point $z$ (since $M$ is bounded, the $L$ is assured in Theorem 1) so that $z=B z+A y$ has a unique solution $z$. Thus, $(I-B) z=A y$ and by the lemma $P y:=(I-B)^{-1} A y$ is a continuous mapping of $M$ into $M$. Now $A M$ is contained in a compact subset of $M$ and $(I-B)^{-1}$ is a continuous mapping of $A M$ into $M$; it is then well known (cf. Kreyszig [4, pp. 412 and 656]) that $(I-B)^{-1} A M$ is contained in a compact subset of $M$. By Schauder's second theorem (cf. Smart [8, p. 25]) there is a fixed point $y=(I-B)^{-1} A y$ or $y=A y+B y$, as required.

Theorem 3. Let $\alpha>0, V, S:[-\alpha, \alpha] \times[-\alpha, \alpha] \rightarrow R, H:[-\alpha, \alpha] \times[-\alpha, \alpha] \times$ $[-\alpha, \alpha] \rightarrow R$ be continuous, and $S(0,0)=V(t, 0)=0$. Suppose that $(X,\|\cdot\|)$ is the Banach space of continuous $\varphi:[-\beta, \beta] \rightarrow R$, with the supremum norm, $0<\beta<\alpha$, $M=\{\varphi \in X \mid \varphi(0)=0,\|\varphi\| \leq \alpha\}$, and that $(B \varphi)(t):=\varphi(t)-V(t, \varphi(t))$ defines a large contraction on $M$. Then (1) has a solution in $M$.

Proof. Note that $B(0)=I(0)-\tilde{V}(0)=0$ where $V(t, \varphi)$ defines a mapping $\tilde{V}$. Next, if $\varphi \in M$ with $\|\varphi\| \leq \alpha / 2$, then $\|B \varphi\|=\|B \varphi-B 0\| \leq\|\varphi-0\| \leq \alpha / 2$. If $\frac{\alpha}{2} \leq\|\varphi\| \leq \alpha$, there exists $\delta<1$ with $\|B \varphi\|=\|B \varphi-B 0\| \leq \delta\|\varphi\| \leq \delta \alpha$. Hence $\|B M\| \leq \max [\delta \alpha, \alpha / 2]=: \gamma<\alpha$.

Now $H$ is continuous on its compact domain, so there is a $K>0$ such that $\varphi \in M$ implies that $|H(t, s, \varphi(s))| \leq K$ for $|t|,|s| \leq \alpha$. Hence, $|t| \leq \beta<\alpha$ and $\varphi \in M \Rightarrow\left|\int_{0}^{t} H(t, s, \varphi(s)) d s\right| \leq K|t| \leq K \beta$. Thus, there is a $\beta>0$ such that $[\varphi \in M,|t| \leq \beta] \Rightarrow\left|S\left(t, \int_{0}^{t} H(t, s, \varphi(s)) d s\right)\right| \leq \alpha-\gamma$.

With this choice of $\beta,[\varphi, \psi \in M,|t| \leq \beta] \Rightarrow\left|S\left(t, \int_{0}^{t} H(t, s, \psi(s)) d s\right)+(B \varphi)(t)\right| \leq$ $\alpha$. If we define a mapping $A: M \rightarrow M$ by $(A \varphi)(t)=S\left(t, \int_{0}^{t} H(t, s, \varphi(s)) d s\right)$, then $\varphi, \psi \in M$ imply $A \varphi+B \psi \in M$.

Now we show that $A$ maps $M$ into a compact subset; in particular, we show that $A M$ is an equicontinuous set. First, $S$ is uniformly continuous, so for a given $\varepsilon>0$ there is a $\delta>0$ such that $\left|t_{1}-t_{2}\right|<\delta$ and

$$
\left|\int_{0}^{t_{1}} H\left(t_{1}, s, \varphi(s)\right) d s-\int_{0}^{t_{2}} H\left(t_{2}, s, \varphi(s)\right) d s\right|<\delta
$$

imply that

$$
\left|S\left(t_{1}, \int_{0}^{t_{1}} H\left(t_{1}, s, \varphi(s)\right) d s\right)-S\left(t_{2}, \int_{0}^{t_{2}} H\left(t_{2}, s, \varphi(s)\right) d s\right)\right|<\varepsilon .
$$


Let $\varphi \in M$ be arbitrary, $\varepsilon>0$ be given, and $\delta$ be the one just mentioned. Suppose that $-\beta \leq t_{1}<t_{2} \leq \beta,\left|t_{2}-t_{1}\right|<\delta$. Then

$$
\begin{aligned}
& \left|\int_{0}^{t_{1}} H\left(t_{1}, s, \varphi(s)\right) d s-\int_{0}^{t_{2}} H\left(t_{2}, s, \varphi(s)\right) d s\right| \\
& \quad=\left|\int_{0}^{t_{1}}\left[H\left(t_{1}, s, \varphi(s)\right)-H\left(t_{2}, s, \varphi(s)\right)\right] d s-\int_{t_{1}}^{t_{2}} H\left(t_{2}, s, \varphi(s)\right) d s\right| \\
& \leq \int_{0}^{t_{1}}\left|H\left(t_{1}, s, \varphi(s)\right)-H\left(t_{2}, s, \varphi(s)\right)\right| d s+\left|t_{2}-t_{1}\right| K .
\end{aligned}
$$

This last quantity can be bounded by $\delta$ if $\left|t_{1}-t_{2}\right|<\delta^{*}$ for some $\delta^{*}>0$ by the uniform continuity of $H$. Hence, $A M$ is equicontinuous. Application of Theorem 2 yields a fixed point $\psi$. Note that $\varphi \in M$ implies $(A \varphi)(0)=0$ and $(B \varphi)(0)=$ $\varphi(0)-V(0, \varphi(0))=0$. Hence, the fixed point $\psi$ satisfies $(1)$ and $\psi(0)=0$. This completes the proof.

Remark. While it is not a corollary, it is still clear from the proof that when $V(t, x)=x$, then the integral equation (1) has a solution under the conditions of Theorem 3 on $H$ and $S$. We simply show that $A$ is a compact map and use Schauder's Theorem.

We now give two related examples for which the classical implicit function theorem fails. The first example utilizes this last remark and fulfills the promise made after (9). The second illustrates verification of a large contraction.

Example 1. Consider the equations $f(t, x)=0, f(0,0)=0$, and suppose that $\frac{\partial f}{\partial x} x^{\prime}+\frac{\partial f}{\partial t}=0$ can be written as $3(x-t)^{2} x^{\prime}=F(t, x)$ with $F(t, x)$ continuous so that the conditions for the implicit function theorem fail at $(0,0)$. We invert the differential operator by writing

$$
\begin{aligned}
-6 \int_{0}^{x}(s-t) d s & +3(x-t)^{2} x^{\prime} \\
& =F(t, x)-3(x-t)^{2}+3 t^{2} \\
& =: G(t, x)
\end{aligned}
$$

or

$$
\frac{d}{d t} \int_{0}^{x} 3(s-t)^{2} d s=G(t, x)
$$

so that

$$
\int_{0}^{x} 3(s-t)^{2} d s=\int_{0}^{t} G(s, x(s)) d s
$$

or

$$
x=t+\left\{-t^{3}+\int_{0}^{t} G(s, x(s)) d s\right\}^{1 / 3},
$$

which is a form of (1) with solution $\varphi(t), \varphi(0)=0$.

Example 2. If $V(x)=x^{3}$ and if $(B \varphi)(t)=\varphi(t)-\varphi^{3}(t)$, then $B$ is a large contraction of the set $M$ of Theorem 3 . 
Proof. In the following computation, $\varphi, \psi$ are evaluated at each $t$. We have $D:=$ $|B \varphi-B \psi|=\left|\varphi-\varphi^{3}-\psi+\psi^{3}\right|=|\varphi-\psi|\left|1-\left(\varphi^{2}+\varphi \psi+\psi^{2}\right)\right|$. Then for

$$
\varepsilon^{2} \leq|\varphi-\psi|^{2}=\varphi^{2}-2 \varphi \psi+\psi^{2} \leq 2\left(\varphi^{2}+\psi^{2}\right)
$$

and for $\varphi^{2}+\psi^{2}<1$ we have

$$
\begin{aligned}
D & \leq|\varphi-\psi|\left[1+|\varphi \psi|-\left(\varphi^{2}+\psi^{2}\right)\right] \\
& \leq|\varphi-\psi|\left[1+\frac{\varphi^{2}+\psi^{2}}{2}-\left(\varphi^{2}+\psi^{2}\right)\right] \\
& =|\varphi-\psi|\left[1-\frac{\varphi^{2}+\psi^{2}}{2}\right] \leq|\varphi-\psi|\left[1-\frac{\varepsilon^{2}}{4}\right]=:|\varphi-\psi| \delta .
\end{aligned}
$$

\section{Concluding Remarks}

The mapping $B$ operates on the closed $\alpha$-ball. Smart lists the open problem [8, p. 39] asking if $B$ a shrinking map will yield a unique fixed point. That is needed in the proof of Theorem 2 where we need to solve $z=B z+A y$. But the crucial point is that the lemma is needed showing $(I-B)^{-1}$ continuous. If $B$ is a shrinking map, then $(I-B)^{-1}$ will exist. But continuity is the big question. Rudin $[5$, p. 78] shows that if the domain is compact and $(I-B)$ is $1-1$, then $(I-B)^{-1}$ is continuous. But we cannot ask that $M$ be compact since the theorem would then be a poor version of Schauder's first theorem. Rudin $[5$, p. 80] gives a counterexample showing that $(I-B)^{-1}$ may be discontinuous if $M$ is not compact. A colleague, R. Kirk, privately communicated a similar example on the closed unit ball in $C[0,1]$ with the supremum norm. In both of these examples $(I-B)$ would lose its 1-1 property if the domain were extended to its closure in some topology. In Kirk's example, the closure was pointwise convergence.

The property of being 1-1 is algebraic, while continuity is topological. A shrinking map $B$ makes $(I-B)$ clearly 1-1. A reasonable conjecture might begin by trying to prove Krasnoselskii's theorem when $B$ is a shrinking map, $M$ is a closed ball, and the closure is taken in a different topology than that of the Banach space.

There is now an enormous amount known about nonexpansive maps. The book edited by Sine [7] contains numerous papers with extensive reference lists. The paper by W.A. Kirk in Sine [7] contains several conditions under which Krasnoselskii's theorem might be proved if continuity of $(I-B)^{-1}$ can be independently verified.

Krasnoselskii's observation that inversion of a perturbed differential operator yields a compact map and a contraction is true in many contexts. Extensions of his result have wide application.

\section{REFERENCES}

1. Corduneanu, C, Integral Equations and Applications, Cambridge Univ. Press, Cambridge, 1991. MR 92h: 45001

2. Hartman, Philip, Ordinary Differential Equations, Wiley, New York, 1973. MR 49:9294

3. Krasnoselskii, M. A., in Amer. Math. Soc. Transl. (2) 10 (1958), 345-409. MR 20:1243

4. Kreyszig, Erwin, Introductory Functional Analysis with Applications, Wiley, New York, 1978. MR 57:7084

5. Rudin, Walter, Principles of Mathematical Analysis, 2nd ed., McGraw-Hill, New York, 1964. MR 29:3587 
6. Schauder, J., Über den Zusammenhang zwischen der Eindeutigkeit und Lösbarkeit partieller Differentialgleichungen zweiter Ordnung von Elliptischen Typus, Math. Ann. 106 (1932), 661-721.

7. Sine, Robert C., Fixed Points and Nonexpansive Mappings, Amer. Math. Soc. (Contemporary Mathematics Vol. 18), Providence, R.I., 1983.

8. Smart, D. R., Fixed Point Theorems, Cambridge Univ. Press, Cambridge, 1980.

9. Taylor, Angus E. and Mann, W. Robert, Advanced Calculus, Third ed., Wiley, New York, 1983. MR 83m:26001

Department of Mathematics, Southern Illinois University, Carbondale, Illinois 62901

E-mail address: taburton@math.siu.edu 